\title{
Plasma concentrations of cholecystokinin, CCK-8, and CCK-33, 39 in rats, determined by a method based on enzyme digestion of gastrin before HPLC and RIA detection of CCK
}

\author{
A LINDÉN, M CARLQUIST, S HANSEN, AND K UVNÄS-MOBERG \\ From the Departments of Pharmacology and Biochemistry II, Karolinska Institute, Stockholm, Sweden and \\ Department of Psychology, University of Gothenburg, Gothenburg, Sweden
}

SUmmary A new specific method for determination of cholecystokinin, CCK-8, and CCK-33, 39 in rat plasma is described. Plasma CCK radioimmunoassay (RIA) is difficult, because of crossreactivity with gastrin. In the rat, problems because of difficulties in separating gastrin from CCK by high performance liquid chromatography (HPLC) exist. These were solved by enzyme digestion of gastrin before HPLC separation of molecular variants of CCK from gastrin fragments. Cholecystokinin immunoreactive forms in the HPLC fractions were determined by an antibody, which recognises the carboxyl terminus of $\mathrm{CCK}$ and gastrin. Fasting concentrations of small (CCK8) and large (CCK-33, 39) molecular forms of CCK averaged 1.9 (0.3) pM and were raised to 13.4 (3.8) $\mathrm{pM}$ in rats fed ad libitum. Cholecystokinin in lactating rats rose two-fold after suckling, compared with $2 \cdot 8$ fold in response to feeding. The basal ratio between CCK-8 and CCK-33, 39 was $\sim 1: 1$, but increased in favour of CCK-8 after feeding and in response to suckling. Gastrin like immunoreactivity measured in unextracted plasma was found to rise after feeding, but was unchanged in response to suckling.

Cholecystokinin (CCK) gives rise to well known gastrointestinal effects, such as increased pancreatic enzyme secretion, gall bladder contraction and inhibition of gastric emptying. ${ }^{2}$ The control of the release of CCK is less well described, however, because of difficulties in measuring CCK in plasma. The major problem with radioimmunoassay (RIA) for CCK, is to obtain CCK specific antibodies that do not crossreact with gastrin, as the biologically active C-terminal tetrapeptide sequence of CCK is structurally identical to gastrin. The very low plasma concentrations of CCK also demand a highly sensitive RIA. Furthermore, the molecular heterogeneity of CCK and gastrin, with structural dissimilarities between species, cause difficulties in the measurement of CCK. There are several studies of plasma

Address for correspondence: Angelica Lindén, MD, Department of Pharmacology, Karolinska Institute, S-104 01 Stockholm, Sweden.

Accepted for publication 15 July 1988.
CCK concentrations in dog and man,,$^{3-x}$ but only few reports on plasma concentrations of CCK in rats as yet. . $^{-11}$

We have previously presented a method for separate determination of CCK- 8 and CCK-33, 39 in dog plasma, based on separation of CCK peptides from gastrin by high performance liquid chromatography (HPLC) before. RIA. ${ }^{12}$ This method was shown to be unsuitable for determination of both CCK-8 and CCK-33, 39 in rat plasma, as rat gastrin unlike dog gastrin, did not elute corresponding to human gastrin standards in the HPLC system. Instead, rat gastrin interfered with the determination of CCK. We have therefore developed a specific method for the determination of CCK- 8 and CCK33,39 in rat plasma, based on enzyme digestion of gastrin before HPLC and consequent RIA detection. In order to measure basal levels, the plasma samples were concentrated 10 -fold before radioimmunoassay. 
Plasma concentrations of CCK are known to increase in response to feeding and various nutrients in the intestine. ${ }^{3-8}$ In previous studies we have described raised concentrations of CCK not only in response to feeding but also during pregnancy and after suckling in lactating dogs. ${ }^{12}{ }^{13}$ In the present study we have measured circulating concentrations of CCK -8 and CCK-33, 39 in fasted, fed and lactating rats. In addition, plasma concentrations of gastrin like immunoreactivity were determined, since we have previously measured increased plasma concentrations of gastrin in response to suckling in dogs. ${ }^{13}$

\section{Methods}

HIGH PERFORMANCE LIQUID CHROMATOGRAPHY Cholecystokinin and gastrin peptides were separated on a TSK ODS-120 T column $(4.6 \times 250 \mathrm{~mm}, 5 \mu \mathrm{m}$ particle size, LKB Produkter, Bromma, Sweden) using an isocratic system of $32 \%$ acetonitrile in $0 \cdot 1 \%$ trifiuoroacetic acid (TFA) $+0 \cdot 155 \mathrm{M} \mathrm{NaCl}$ with a flow rate of $1 \mathrm{ml} / \mathrm{min}$. This HPLC system resulted in a good separation of CCK-8 and CCK-33, 39, nonsulphated and sulphated gastrin-17 and gastrin-34 (Fig. 1). Standards used were sulphated (s) CCK-8, human non-sulphated (ns) gastrin-34, and human non-sulphated and sulphated gastrin-17 (Cambridge Research Biochemicals, Harston, Cambridgeshire, England) and porcine sulphated CCK-33 and CCK-39 (gifts from Professor V Mutt, Stockholm, Sweden).

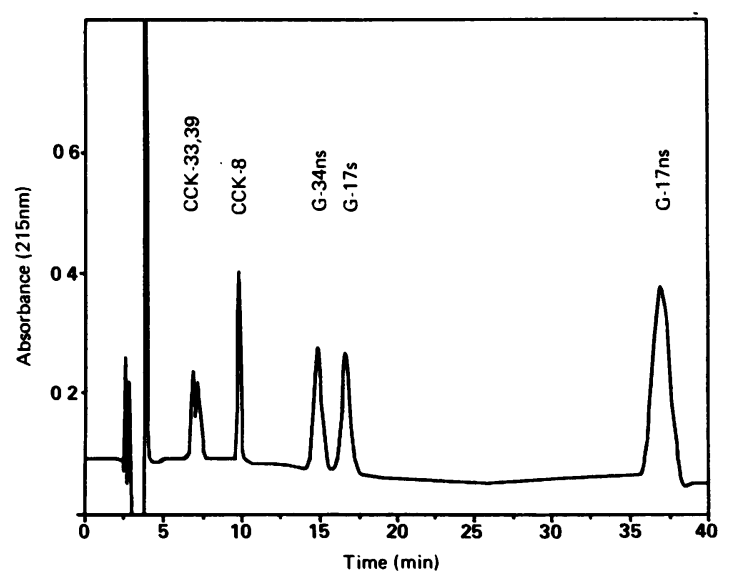

Fig. 1 Separation of CCK-8 (s), porcine CCK-33 (s), CCK-39 (s), human gastrin-17 (ns and s), and human gastrin-34 (ns) by reversed-phase HPLC. Column: TSK ODS-120 T (4.6×250 mm, $5 \mu \mathrm{m}$ particle size). Solvent system: $32 \%$ acetonitrile in $0.1 \% \mathrm{TFA}+0.155 \mathrm{M} \mathrm{NaCl}$. Flow rate $1 \mathrm{ml} / \mathrm{min}$.
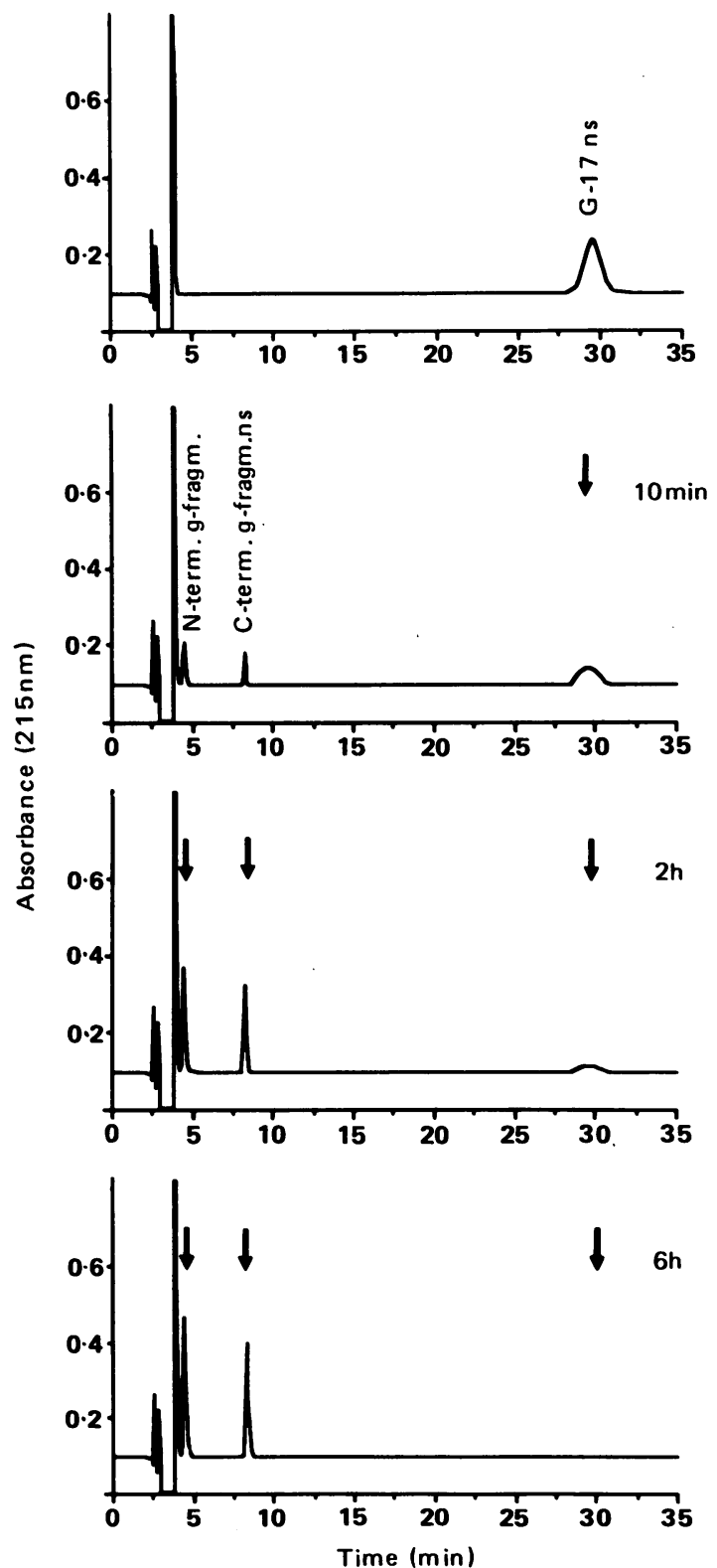

Fig. 2 Human gastrin-17 ( $\mathrm{ns}$ ) before and after a $10 \mathrm{~min}$, two hours, and six hours long incubation period $\left(\mathrm{pH} \mathrm{8.0,37^{ \circ } )}\right.$ with protease from $\mathrm{S}$ aureus, detected by $H P L C$. The occurrence of the N-terminal (1-6) and C-terminal (11-17) gastrin fragment are indicated. For details about column and solvent system, see legend to Fig. 1.

ENZYME DIGESTION

Protease (Staphylococcus aureus V.8, Miles Scientific, Naperville, IL, U.S.A.), an enzyme which 
under certain conditions specifically cleaves peptide bonds on the $\mathrm{COOH}$ terminal side of glutamic acid ${ }^{14}$ will cleave gastrin but not $\mathrm{CCK}$, as CCK peptides do not contain glutamic acid whereas gastrins do. Enzyme digestion of gastrin-17 (ns) detected by HPLC at 10 min, 1, 2, 4, 6, 8, 12, and 24 hours results in the formation of two peaks which reach their maximum size after a six hour incubation period (Fig. $2)$. Digestion was performed in $1 \%$ ammonium bicarbonate $(w / v), \mathrm{pH} 8 \cdot 0$, at $37^{\circ} \mathrm{C}$ with an enzyme/ substrate ratio of $1: 10(\mathrm{w} / \mathrm{w})$. High performance liquid chromatography fractions corresponding to peak 1 and 2 were collected, analysed after acid hydrolysis and identified as the N-terminal $(1-6)$ and C-terminal (11-17) fragment of gastrin-17, respectively (Table). Enzyme digestion of gastrin-34 (ns) and gastrin-17 (s) resulted in N-terminal and Cterminal fragments which coeluted with the respective fragments derived from gastrin-17 (ns), except C-terminal fragments from gastrin-17 (s) which eluted earlier. High performance liquid chromatography separation of CCK and gastrin standards after enzyme digestion is shown in Figure 3.

GEL FILTRATION

Lyophilised tissue extracts with and without prior

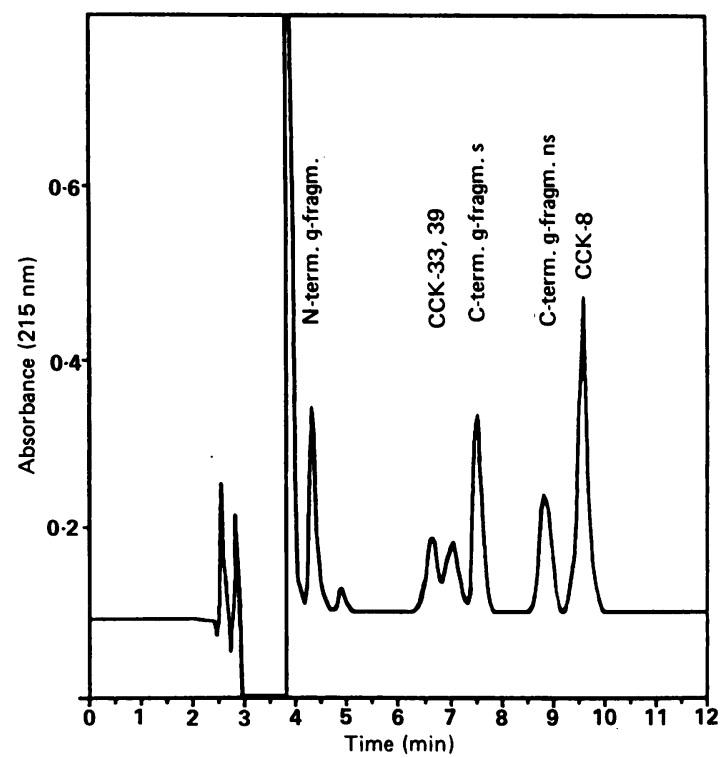

Fig. 3 Enzyme digestion of CCK-8 (s), porcine CCK-33 (s), CCK-39 (s), human gastrin-17 ( $n$ s and s), and human

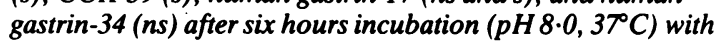
protease from $S$ aureus. The figure shows the formation of the $N$-terminal gastrin fragment and the nonsulphated and sulphated C-terminal gastrin fragment, detected by HPLC. For details about column and solvent system, see legend to Fig.1.
Table Amino acid compositions of fragments obtained after cleavage of gastrin-17 with the protease from Staphylococcus aureus $V .8$

\begin{tabular}{lll}
\hline Aminoacid & Peak 1(1-6) & Peak 2(11-17) \\
\hline Trp & nd (1) & nd (1) \\
Asp & & $1 \cdot 0(1)$ \\
Glu & $1.9(2)$ & \\
Pro & $1.0(1)$ & \\
Gly & $1 \cdot 2(1)$ & $1 \cdot 1(1)$ \\
Ala & & $1 \cdot 0(1)$ \\
Met & $0.9(1)$ & $0 \cdot 9(1)$ \\
Leu & & $1 \cdot 0(1)$ \\
Tyr & & $1 \cdot 0(1)$ \\
Phe & 6 & 7 \\
Total & & \\
\hline
\end{tabular}

Values are molar ratios without correction for destruction, incomplete hydrolysis, or impurity. nd: not detected.

enzyme digestion were reconstituted in $2 \mathrm{ml}$ Veronal buffer (see below) and applied to a Sephadex G-50 superfine column $(16 \times 1000 \mathrm{~mm})$ eluted with $0.02 \mathrm{M}$ Veronal $+0 \cdot 1 \%$ BSA (w/v), $\mathrm{pH} 8 \cdot 4$, at $4^{\circ} \mathrm{C}$ at a flow rate of $5 \mathrm{ml} / \mathrm{h}$. The column was calibrated with Blue Dextran (for indication of void volume, Vo), Gastrin-34 (ns), CCK-39, CCK-33, gastrin-17 (ns), CCK-8, and ${ }^{125}$. Fractions of $1 \mathrm{ml}$ were collected for analysis by radioimmunoassay.

\section{RADIOIMMUNOASSAYS}

Cholecystokinin concentrations were determined by gastrin radioimmunoassay ${ }_{1}^{15}$ with the modification of using antiserum no 2609 (gift from Professor J F Rehfeld, Copenhagen, Denmark), which reacts with the biologically active C-terminal tetrapeptide common to both gastrin and $\mathrm{CCK}^{16}$ and by using sulphated CCK-8 as standard. Antiserum no 2609 will be referred to as the carboxyl-terminal antiserum in the following text. Standard curves were made in the assay buffer $(0.02 \mathrm{M}$ Veronal) with the same salt concentration as in the unknown samples after HPLC fractionation. The intra-assay coefficient of variation is $3-5 \%$, the interassay coefficient is between $8-12 \%$, and the sensitivity of the assay if $5 \mathrm{pM}$. Molar crossreactivity of CCK-8:33:39 is 1:0.75:0.75 at ID 50. No corrections for the differences in binding activity between CCK peptides have been done.

Specific gastrin immunoreactivity was measured by radioimmunoassay ${ }^{15}$ using antiserum no 2604 (gift from Professor J F Rehfeld). This antiserum binds the C-terminal hexapeptide of gastrin-17 and recognises both sulphated and non-sulphated forms of gastrin as well as gastrin- 17 and -34 with the same potency. The sensitivity of the assay is $5 \mathrm{pM}$ and this antiserum is highly specific for gastrin because the cross reactivity with CCK is negligible (ratio of ID 50 for gastrin-17 to ID 50 for CCK $<0.001)$. ${ }^{17}$ 
When plasma samples after HPLC fractionation were determined by both the unspecific carboxylterminal antiserum 2609 and the gastrin specific antiserum 2604, no gastrin specific immunoreactivity was found in fractions corresponding to standards of CCK-8 and CCK-33, 39. Thus, in this study, immunoreactivity found with the carboxyl-terminal assay in fractions corresponding to CCK standards, is considered as CCK. Furthermore, gastrin was determined in unextracted plasma samples using antiserum 2604.

TREATMENT OF TISSUE BIOPSIES

Tissue biopsies of the antrum and duodenum were taken from two rats directly after decapitation. The mucosa was stripped off, immediately put on dry ice and kept frozen until extraction was carried out. Antral mucosa $(\sim 0.40 \mathrm{~g} / \mathrm{rat})$ was pooled from the two rats and boiled in water $(10 \mathrm{ml} / \mathrm{g}$ of tissue, $\mathrm{pH} 6.6)$ for 10 minutes. Human serum albumin (HSA) $0.1 \%$ w/v, was added and the suspension was centrifuged at $2000 \mathrm{rpm}$ for 10 minutes. The supernatant was decanted and divided into two samples before lyophilisation. The duodenal mucosa $(\sim 0.2 \mathrm{~g} / \mathrm{rat})$ from the two rats was pooled, and first extracted at neutral $\mathrm{pH}$ (as described above) and then was $0.1 \mathrm{M} \mathrm{HCl}(10 \mathrm{ml} / \mathrm{g}$ of tissue, $\mathrm{pH} \mathrm{1.5)}$ added to the insoluble material. ${ }^{18}$ The suspension was centrifuged and the supernatant divided into two samples before lyophilisation. One of the duplicate samples from antral and duodenal mucosa, respectively, was enzyme treated with protease from $S$ aureus and then lyophilised before gel filtration.

TREATMENT OF PLASMA SAMPLES Plasma was purified on C18 SEP-PAK cartridges (Waters Associates, Milford, MA, USA). The cartridges were equilibrated with $10 \mathrm{ml} 100 \%$ acetonitrile $(\mathrm{v} / \mathrm{v})$, followed by $10 \mathrm{ml} 0 \cdot 1 \%$ acetic acid $(\mathrm{v} / \mathrm{v})$ before the application of $2 \mathrm{ml}$ plasma samples. After washing with $5 \mathrm{ml} 0 \cdot 1 \%$ acetic acid, the samples were eluted by $6 \mathrm{ml}$ of acetonitrile and $0 \cdot 1 \%$ acetic acid $(1: 1, v / v)$ and then lyophilised.

The lyophilised samples were reconstituted in 200 $\mu \mathrm{l}$ of $1 \%$ ammonium bicarbonate (w/v, $\mathrm{pH} 8.0$ ) before a six hour incubation period $\left(37^{\circ} \mathrm{C}\right)$ with protease from $S$ aureus $(5 \mu \mathrm{g} / \mathrm{sample})$. After the enzyme digestion the samples were again lyophilised.

Enzyme-treated samples were dissolved $(500 \mu \mathrm{l}$ $0 \cdot 1 \%$ TFA and water, $1: 1, \mathrm{v} / \mathrm{v})$, filtrated through a Millex-HV filter (Millipore Corporation, Bedford, MA, USA) before loading onto the HPLC column for peptide separation using the system described above. Fractions $(0.25 \mathrm{ml})$ were collected, frozen and lyophilised before analysis by radioimmunoassay.

The lyophilised samples were reconstituted in
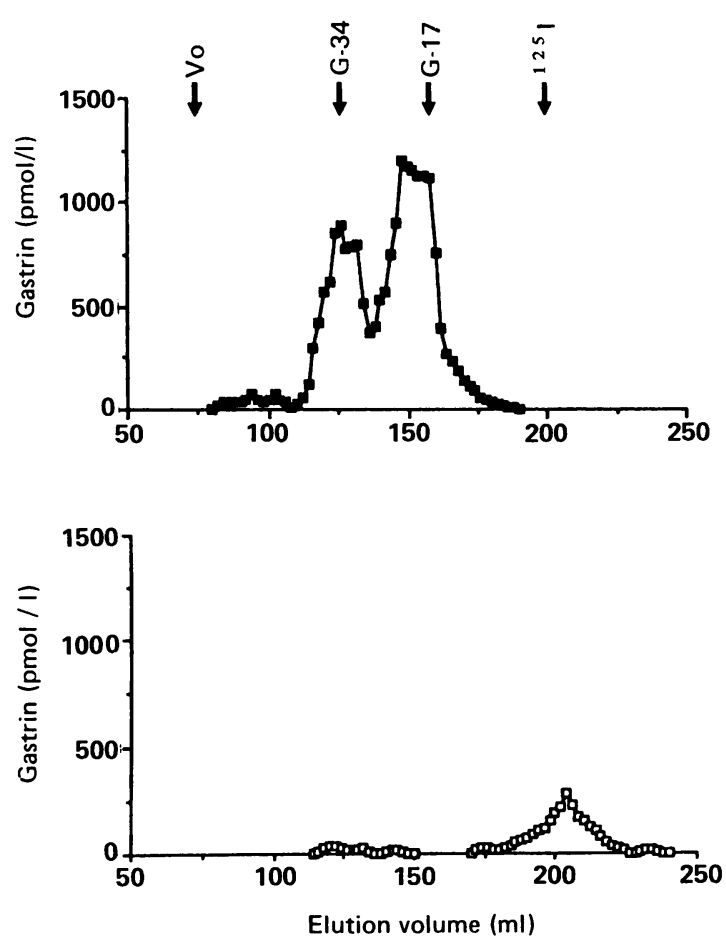

Fig. 4 Gel chromatography of rat antral extract, (a) nontreated extract ( $(\mathbf{)}$ ) and (b) enzyme treated extract ( $\square$ ). Column: Sephadex G-50 (superfine, $16 \times 1000 \mathrm{~mm}$ ). Column buffer: $0.02 \mathrm{M}$ Veronal $+0.1 \% \mathrm{BSA}, \mathrm{pH} 8.4$. Flow rate $5 \mathrm{ml} / \mathrm{h}$. Fractions of $1 \mathrm{ml}$ were collected and assayed with a gastrin specific radioimmunoassay using antiserum 2604. The column was calibrated with Blue Dextran (for indication of void volume, Vo), human gastrin-34 (ns), porcine CCK-39(s), and CCK-33 (s), human gastrin-17(ns), $C C K-8(s)$, and ${ }^{125} I$.

$200 \mu \mathrm{l}$ of the assay buffer (0.02 M Veronal). Cholecystokinin immunoreactivity was determined by the carboxyl-terminal antiserum. Gastrin specific immunoreactivity was also determined for detection of C-terminal gastrin fragments and to control that no gastrin was present in fractions corresponding to CCK standards.

\section{RAT EXPERIMENTS}

Female virgin and lactating Wistar rats weighing approximately $250 \mathrm{~g}$ were used in the experiments.

\section{FASTING AND FEEDING}

Control rats

One group of rats was deprived of food for $48 \mathrm{~h}$ (water was freely available), before decapitation when trunk blood was collected. Another group of rats had ordinary food and water ad libitum before decapitation. 
Vagotomised rats

Two groups of rats, anaesthetised with Ketalar, underwent a bilateral truncal vagotomy $48 \mathrm{~h}$ before use. One group of vagotomised rats was starved for $48 \mathrm{~h}$ (water was freely available) and the other group had food and water ad libitum before decapitation.

SUCKLING EXPERIMENTS IN LACTATING RATS Lactating rats with 10 pups each, were starved and separated from their pups $13 \mathrm{~h}$ before the experiment was performed.

In two groups of rats, pups were returned to their mothers. In one of the groups, lactating rats were decapitated two minutes after start of suckling and in the other group after $\sim 10$ min of suckling when milkejection occurred, as indicated by a characteristic behaviour, the stretch reaction. A separate group of rats was offered food pellets and was allowed to eat for two minutes before decapitation. Control rats were decapitated after the fasting and separation period.

COLLECTION OF SAMPLES

Trunk blood $(\sim 5 \mathrm{ml})$ was collected in ice chilled tubes containing 10 IU heparin/ml (Kabi AB, Stockholm, Sweden) and $500 \mathrm{IU}$ aprotinin $/ \mathrm{ml}$ (Bayer AB, Stockholm, Sweden). The samples were immediately centrifuged and plasma stored at $-20^{\circ} \mathrm{C}$ until assayed for CCK and gastrin.

STATISTICAL ANALYSIS

Data are presented as mean (SEM). Comparisons between groups were evaluated with the KruskalWallis one-way analysis of variance and subsequent two group comparisons were made with the MannWhitney U test, provided the analysis of variance first showed a significant overall effect. The value of $p<0.05$ is considered as significant for a two-tailed test. There were five to seven rats in each group.

\section{Results}

GASTRIN AND CCK IN RAT ANTRAL AND

DUODENAL MUCOSA

Gel chromatography of antral extract produced two peaks of gastrin immunoreactivity. One peak eluted in the same area as gastrin-34 and the other peak eluted in correspondence with gastrin-17 (Fig. 4a). The gel filtration pattern of duodenal extract revealed the presence of two or three CCK immunoreactive peaks, which eluted in the same area as CCK-39 and CCK-33 (which coelute) and corresponding to CCK-8 (Fig. 5a).

Gel chromatography of enzyme treated antral extract revealed a completely changed elution pattern, whereas the gel filtration pattern of duo-
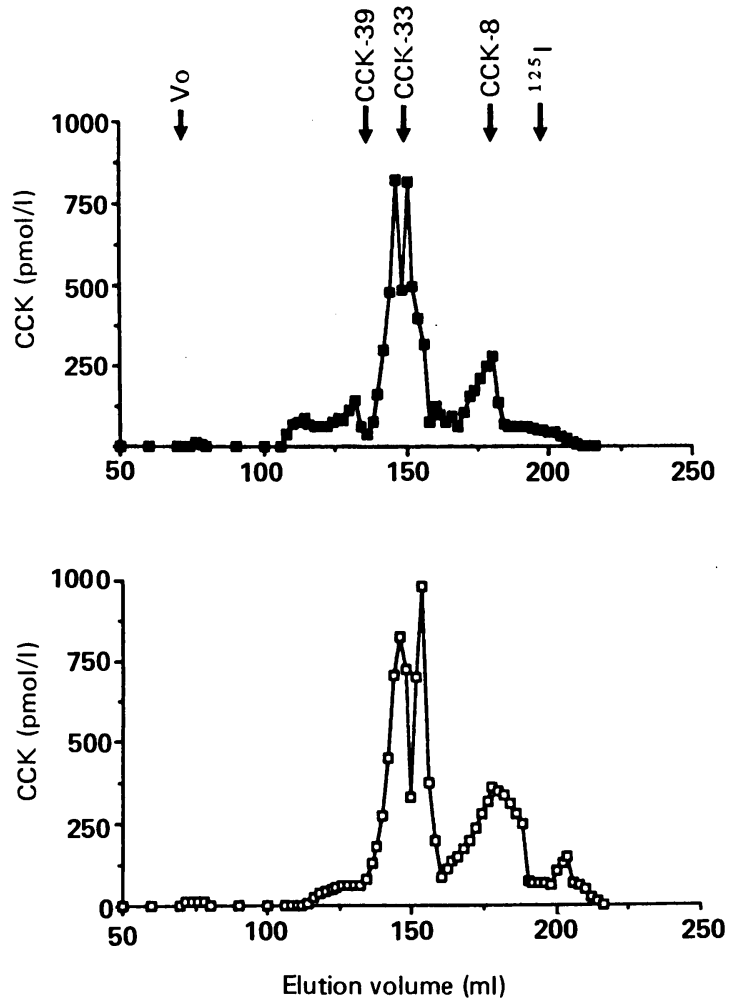

Fig. 5 Gel chromatography of rat duodenal extract, (a) non-treated extract ( $(\mathbf{)}$ ) and (b). enzyme treated extract ( $\square$ ). Fractions were assayed for CCK immunoreactivity with carboxyl-terminal antiserum 2609. For details about column and methods, see legend to Fig. 4.

denal extract was not changed by enzyme treatment (Figs $4 b$ and $5 b$ ).

When rat gastrin-17 and -34 obtained after gel filtration of antral mucosa were applied to the HPLC column, it was shown that the elution positions did not correspond to the respective human gastrin standards, since rat gastrin eluted considerably earlier in fractions that also contain CCK (Fig. 6a). Cholecystokinin immunoreactive material obtained after gel filtration of rat duodenal extract corresponding to standards of CCK-8 and CCK-33, 39, however, was shown to coelute with the CCK standards on the HPLC column (Fig. 6b). It is therefore likely that CCK immunoreactive material obtained from the rat, eluting in the same positions as CCK standards (synthetic CCK-8, porcine CCK-33, 39) after HPLC chromatography corresponds to rat CCK-8 and CCK-33, 39.

CCK AND GASTRIN IN RAT PLASMA

A plasma sample from a fed rat was divided into two samples and one of the duplicates was subjected to 

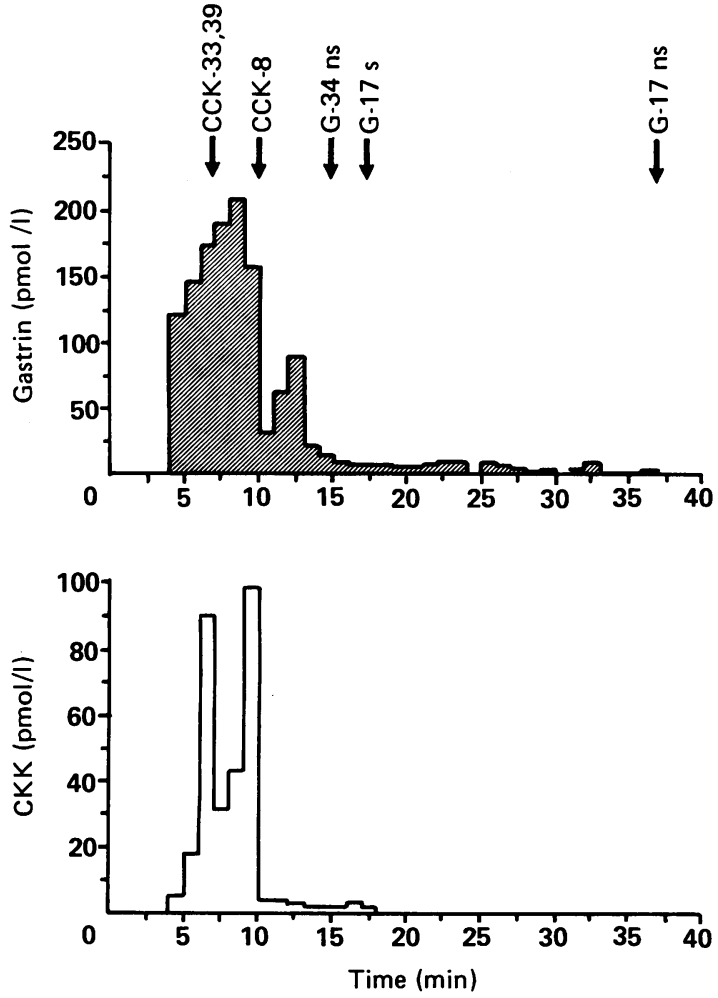

Fig. 6 Immunoreactivity of (a) rat gastrin and (b) rat CCK (obtained following gel filtration of antral and duodenal extract), detected in HPLC fractions assayed for gastrin and CCK immunoreactivity with gastrin specific antiserum 2604 and carboxyl-terminal antiserum 2609 , respectively. For details about HPLC column and solvent system, see legend to Fig. 1. Positions for CCK-8 (s), CCK-33 (s), CCK-39 (s), gastrin-17 (ns and s), and gastrin-34 (ns) are indicated.

enzyme digestion of gastrin like peptides before application to the HPLC system. The profile of cholecystokinin and gastrin like immunoreactivity of the non-treated and enzyme treated plasma sample is shown in Figs 7a and 7b, respectively. In the enzymetreated plasma sample, immunoreactive material was found with the carboxyl terminal assay, but not with the gastrin specific assay in fractions corresponding to exogenous CCK-8 (elution time $9.5-10.5 \mathrm{~min}$ ) and CCK-33 and -39 (which coelute, elution time 6.25$7.25 \mathrm{~min}$ ). These fractions are therefore considered to contain rat CCK. This is in contrast with the nontreated plasma sample, which contained gastrin specific immunoreactivity in the fractions for CCK-8 and CCK-33, 39. Furthermore, in the enzymetreated plasma sample immunoreactivity was found with the carboxyl-terminal assay and the gastrin specific assay in fractions corresponding to Cterminal fragments of non-sulphated and sulphated human gastrin-17 and -34 (9 and $7.5 \mathrm{~min}$, respectively).

Measurement of low concentrations of CCK was made possible by a 10 -fold concentration of the $2 \mathrm{ml}$ plasma sample, which was processed as described above and then reconstituted in $200 \mu$ l of assay buffer before radioimmunoassay. Recovery studies of CCK-8 and CCK-33 without and after enzyme digestion, in amounts ranging from 0.02 to 0.2 pmol added to $1 \mathrm{ml}$ rat plasma and processed as above, revealed that the CCK content was unaffected by enzyme treatment and $50 \pm 4 \%$ of the original material was recovered.

\section{RAT EXPERIMENTS: FASTING AND FEEDING CCK}

Total CCK concentrations (CCK-8+CCK-33,39) in the group of fasted control rats were $1.9(0.3) \mathrm{pM}$ and were significantly raised $(\mathrm{p}<0.05)$ to $13.4(3.8) \mathrm{pM}$ in the group of freely fed rats. In the group of vagotomised fasted rats, total CCK concentrations were 3.7 $(0.3) \mathrm{pM}$, which is significantly higher $(\mathrm{p}<0.05)$ than in fasted control rats. Cholecystokinin concentrations in fed vagotomised rats averaged $1 \cdot 1(0 \cdot 4) \mathrm{pM}$, which is significantly lower $(\mathrm{p}<0.05)$ than in fasted vagotomised rats. When the CCK like immunoreactivity appearing in fractions corresponding to CCK-8 and CCK-33, 39 were calculated separately, it was found that the ratio between small (CCK-8) and large (CCK-33, 39) molecular types differed among the groups. Thus, the ratio CCK-8/CCK-33, 39 in the group of fasted control rats was $\sim 1: 1$ but increased in favour of CCK-8 in the group of fed control rats. In the group of fed vagotomised rats, however, the ratio CCK-8/CCK-33, 39 differed significantly $(\mathrm{p}<0.05)$ to be in favour of CCK-33, 39. In the group of fasted vagotomised rats, CCK-8 was the predominant form. Fig. 8a.

\section{Gastrin}

Gastrin concentrations averaged $51(4 \cdot 1) \mathrm{pM}$ in fasted control rats and were significantly raised $(\mathrm{p}<0.01)$ to $120(12.6) \mathrm{pM}$ in fed control rats. Gastrin concentrations in the group of fasted vagotomised rats were $83(7.3) \mathrm{pM}$, which is significantly higher $(\mathrm{p}<0.05)$ than in the group of fasted control rats. In the group of fed vagotomised rats, gastrin concentrations averaged $63(4 \cdot 5) \mathrm{pM}$. Fig. $8 \mathrm{~b}$.

\section{SUCKLING EXPERIMENTS} CCK

Total CCK concentrations were 6.9 (1) $\mathrm{pM}$ in lactating control rats, $13.5(1.5) \mathrm{pM}$ in the suckling group, $10 \cdot 2(0.7) \mathrm{pM}$ in the milk ejection group and $19.5(3.8) \mathrm{pM}$ in the fed group. The concentrations of CCK were significantly raised $(\mathrm{p}<0.05)$ in all experi- 


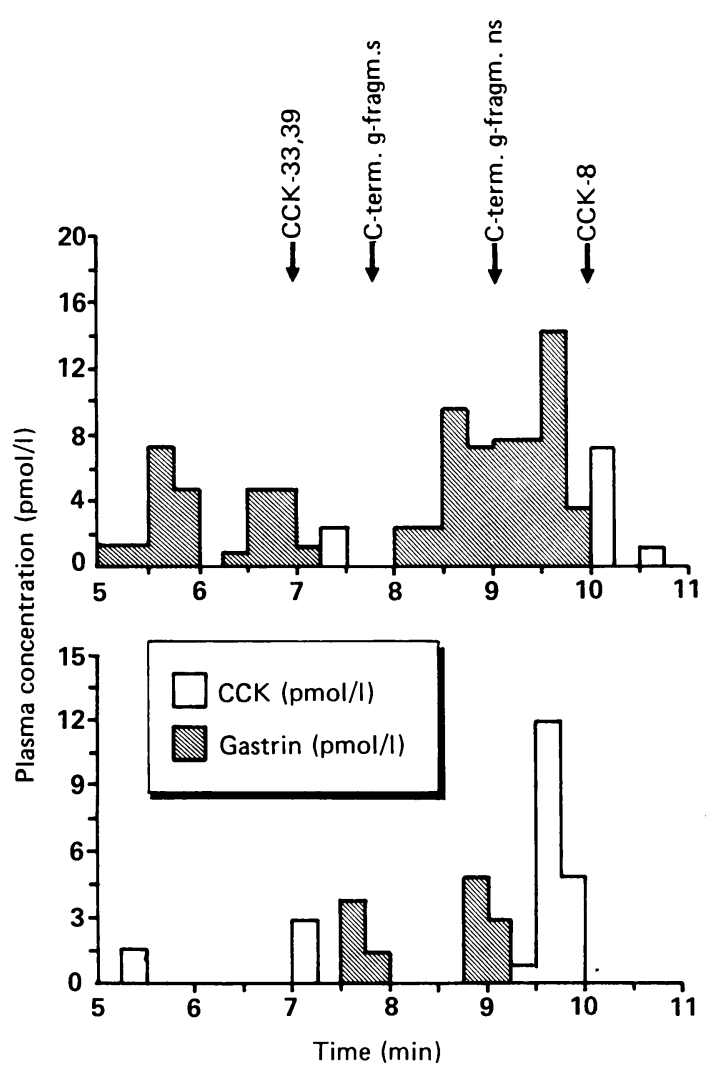

Fig. 7 Cholecystokinin and gastrin immunoreactivity in rat plasma after feeding, (a) non-treated sample and (b) enzyme treated sample, detected in HPLC fractions by carboxylterminal antiserum 2609 and gastrin specific antiserum 2604. Fractions containing immunoreactivity exclusively found by the carboxyl-terminal assay (considered as CCK) is marked by open bars, and fractions containing gastrin specific immunoreactivity is marked by cross striped bars. For details about HPLC column and solvent system, see legend to Fig. 1 . Positions for CCK-8 (s), CCK-33 (s), CCK-39 (s), and nonsulphated and sulphated $C$-terminal gastrin fragments are indicated.

mental groups compared with CCK concentrations in the control group. The ratio CCK-8/CCK-33, 39 was in favour of CCK-33, 39 in the control group but was significantly changed $(\mathrm{p}<0.05)$ to be increased in favour of CCK-8 in the fed group and with the same tendency in the suckling group $(p=0 \cdot 06)$. Fig. 9a.

\section{Gastrin}

Gastrin concentrations averaged $49(6 \cdot 3) \mathrm{pM}$ in the control group, $68(12 \cdot 8) \mathrm{pM}, 66(11 \cdot 1) \mathrm{pM}$, and 182 $(31 \cdot 1) \mathrm{pM}$ in the suckling, milk ejection and fed group, respectively. Only gastrin concentrations in the fed group were significantly raised $(p<0 \cdot 05)$
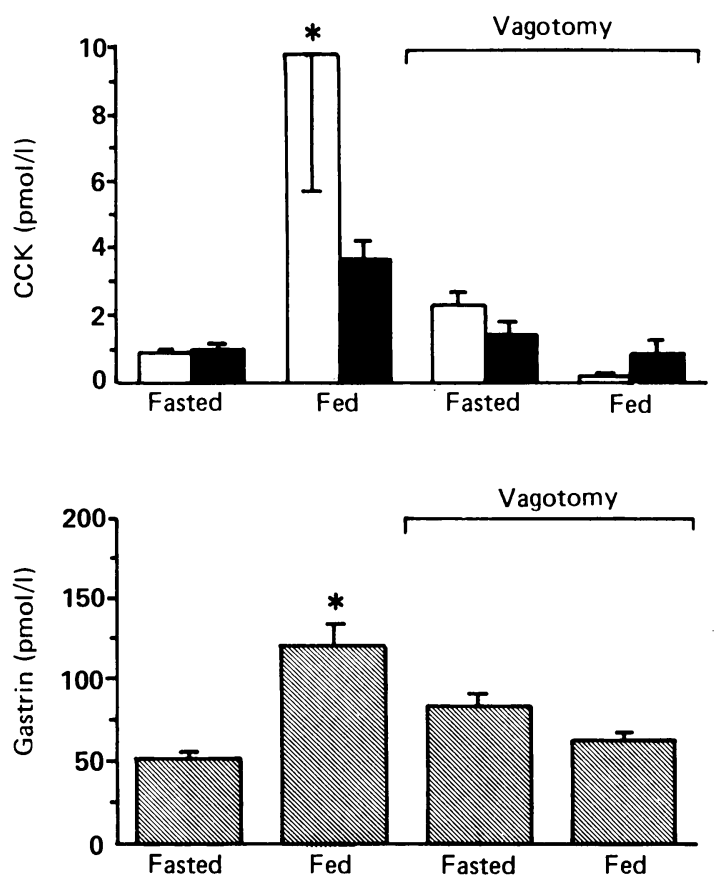

Fig. 8 Average plasma concentrations of (a) $C C K-8$ (white bars), CCK-33, 39 (black bars) (b) gastrin (SEM) in fasted and fed control and vagotomised rats. Total $C C K$ concentrations $(C C K-8+C C K-33,39)$ differed significantly $(p<0.05)$ between the fasted and fed control group (indicated by $\star)$, the fasted and fed vagotomised group and between the fasted control and fasted vagotomised group of rats. The ratio CCK-8/CCK-33, 39 differed significantly $(p<0 \cdot 05)$ between the fed control and fed vagotomised group and between the fasted and fed vagotomised group of rats. Gastrin concentrations were significantly $(p<0.01)$ higher in the fed control group (indicated by $\star$ ) compared with the fasted control group and in the fasted vagotomised group compared with the fasted control group of rats $(p<0 \cdot 05)$.

compared with gastrin concentrations in the control group. Fig. 9b.

\section{Discussion}

CCK DETERMINATION IN RAT PLASMA

We have previously developed a method for the determination of CCK-8 and CCK-33, 39 in dog plasma, based on HPLC separation of CCK from gastrin before radioimmunoassay. ${ }^{12}$ This method was not acceptable for CCK measurements in rat plasma, as specific gastrin immunoreactivity appeared in HPLC fractions for CCK-33, 39. Further investigations showed, that rat gastrin-17 and - 34 (material collected from gel filtration of rat antrum extract), in contrast with dog gastrin, did not elute corresponding to the respective human gastrin standards in our 

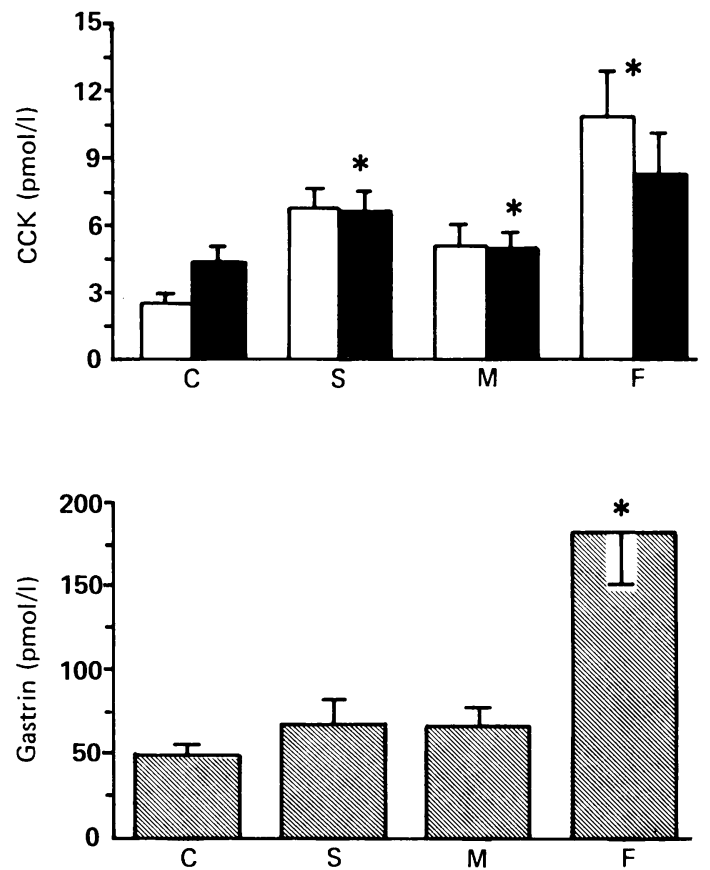

Fig. 9 Average plasma concentrations of (a) $C C K-8$ (white bars), CCK-33, 39 (black bars) (b) gastrin (SEM) in a lactating control group $(C)$, suckling group $(S)$, milk ejection group $(M)$, and in a group of fed lactating rats $(F)$. Total CCK concentrations were significantly $(p<0.05)$ raised in all experimental groups (indicated by $\star$ ) compared with the control group. The ratio $C C K-8 / C C K-33,39$ differed significantly $(p<0.05)$ between the fed and the control group. In the suckling group there was a tendency of changed ratio $(p=0 \cdot 06)$ compared with the control group. Gastrin concentrations in the fed group were significantly $(p<0 \cdot 01)$ raised compared with the control group (indicated by $\star$ ).

HPLC system. Instead, rat gastrin eluted much earlier in fractions also containing CCK.

It has previously been shown that rat gastrin differs from other mammalian gastrins in certain physical and chemical properties. ${ }^{1920}$ Thus, rat gastrin-17 elutes before human and porcine gastrin-17 from Sephadex G 50 columns, and it elutes earlier than human gastrin-17 from anion exchange columns. Furthermore, rat gastrin-17 has poor immunoreactivity with antibodies specific for the $\mathrm{N}$-terminal region of human of porcine gastrin-17 but good immunoreactivity with antibodies specific for the C-terminal region of gastrin-17. The different behaviours of rat gastrin as described above can be explained by the amino acid sequence for rat gastrin, which substantially differs from that of other species at the amino terminus of gastrin-17. The sequence,
Gln-Gly-Pro-Trp described for all other gastrins sequenced $^{21}$ is Gln-Arg-Pro-Pro in rat gastrin. ${ }^{2022}$ This variance is probably the reason for the different elution positions of rat gastrin compared to human gastrin standards in chromatographic systems.

We solved the problem of incomplete separation of CCK from gastrin in our HPLC system by using an enzyme which cleaves gastrin but not CCK, before HPLC separation and RIA detection. The enzyme is a protease from Staphylococcus aureus V.8, which specifically cleaves peptide bonds on the $\mathrm{COOH}$ terminal side of glutamic acid when digestion is carried out in ammonium bicarbonate or ammonium acetate buffer ${ }^{14}$ Since in this study, the digestion was done in ammonium bicarbonate buffer, and CCK peptides do not contain glutamic acid, no cleavage of CCK was seen. In contrast, gastrin which contains glutamyl bonds was digested. By using enzymatic cleavage of gastrin, we obtained a complete HPLC separation of CCK peptides from all gastrin like peptides. The method was controlled by using two antibodies, a carboxyl-terminal ab (2609), which detects both CCK and gastrin, and a gastrin specific ab (2604). After enzyme digestion, no gastrin specific immunoreactivity was found in fractions corresponding to CCK standards. Thus, immunoreactivity found by the carboxyl-terminal assay in those fractions, is considered as CCK. Therefore, no substraction of results obtained by ab 2609 from that of ab 2604 was made.

MOLECULAR VARIANTS OF CCK IN THERAT There is a considerable molecular heterogeneity described for intestinal CCK. ${ }^{62-25}$ Cholecystokinin-8 and CCK-33, 39, are, however, the intestinal molecular variants that are common to most species. Cholecystokinin-58 has recently been reported to be the most abundant form in dog intestine. ${ }^{2 h}$ In the rat, intestinal CCK-22 has been demonstrated. ${ }^{27}$ It should be mentioned that not only do the pattern of CCK variants differ between species, the type of extraction method used influences the pattern as well. Thus smaller molecular forms of CCK are better extracted at neutral $\mathrm{pH}$, whereas larger forms of CCK are more efficiently extracted at acidic $\mathrm{pH} .{ }^{18}$ In order to obtain maximal extraction of both small and large molecular forms of CCK, we have in this study combined neutral and acid extraction.

In the present study, Sephadex G-50 chromatography of rat duodenal extract showed CCK immunoreactive peaks corresponding to porcine CCK-33, 39 and synthetic CCK-8. Material from the CCK immunoreactive peaks obtained from gel chromatography of rat duodenal extract was applied onto the HPLC column for separation. The CCK immunoreactive peaks corresponding to porcine 
CCK-33, 39 and synthetic CCK-8 eluted in the same positions as the CCK standards in our HPLC system. Therefore, we suggest that the CCK immunoreactivity obtained from rat tissue and rat plasma that elutes in HPLC fractions for standards of CCK-8 and CCK-33, 39 may correspond to rat CCK-8 and rat CCK-33, 39. As CCK-22 has been reported to occur in rat intestine, however, we can not exclude that CCK-22 is included in the immunoreactive peaks corresponding to porcine CCK-33, 39.

\section{CCK CONCENTRATIONS AND MOLECULAR}

VARIANTS IN RAT PLASMA

With the present method, which made it possible to determine CCK-8 and CCK-33, 39 separately, basal levels of CCK (CCK-8+CCK-33, 39) in female virgin rats were found to be low (1.9 pM). Measurement of this low concentration of CCK was made possible by a 10-fold concentration of the $2 \mathrm{ml}$ plasma sample, processed as described above and then reconstituted in $200 \mu \mathrm{l}$ before radioimmunoassay. In the group of rats fed ad libitum, cholecystokinin concentrations were many times increased to $13.4 \mathrm{pM}$. Low basal and postprandial concentrations of CCK are in agreement with most studies in dog and $\operatorname{man}^{3568}$ and the few reported in rat by radioimmunoassay and bioassay. ${ }^{10}{ }^{11}$ In a previous study of ours, in which only concentrations of CCK-8 were measured in rat plasma, the levels were found to be in line with the present ones. ${ }^{2 x}$

The different molecular forms of CCK in plasma have been studied in dog and man..$^{356}$ Cholecystokinin-8 and CCK-33, 39 have been reported to be the most abundant forms in plasma until recently, when CCK- 58 has been claimed to be a major type of CCK in dog and human plasma. ${ }^{29}{ }^{30}$ In the rat, two forms of circulating CCK have been described (by gel filtration in combination with bioassay), one peak of intermediate size between CCK-33 and CCK-8 and another peak with the same size as CCK-8. " By the present method, two types of CCK are detected, one corresponding to CCK-8 and one to CCK-33, 39. We cannot, however, exclude that the CCK immunoreactivity in HPLC fractions for porcine CCK-33, 39 may also contain CCK-22.

\section{EXPERIMENTAL RESULTS}

Because 1-2 ml plasma is needed for CCK determination with the present method, the rats were decapitated for collection of trunk blood. It is not excluded that decapitation per se induces a stress reaction which may influence the release of gastrointestinal hormones, but the basal gastrin concentrations recorded in this study do not seem to be raised when compared with other studies. ${ }^{31}$

Plasma concentrations of both CCK (CCK-8+
CCK-33, 39) and gastrin were increased in freely fed rats compared with fasted rats. Raised plasma concentrations of gastrin in response to feeding have previously been shown in rats. ${ }^{32}$ The ratio between CCK-8 and CCK-33, 39 was $\sim 1: 1$ in fasting samples, but CCK-8 was in majority in samples from fed rats. This finding may be explained by a relatively larger release of CCK-8 compared to CCK-33, 39 in response to feeding. We have previously shown that CCK-8 is released in majority into plasma compared to CCK-33, 39 immediately after feeding in dogs. ${ }^{12}$

In the vagotomised rats, basal CCK concentrations were significantly increased compared with control rats, whereas plasma concentrations of CCK were decreased in the group of fed vagotomised rats. Interestingly, the ratio $\mathrm{CCK} / 8-\mathrm{CCK}-33,39$ was inverted in samples of fed vagotomised rats compared with fed control rats. Gastrin concentrations were also raised in fasted vagotomised rats. The reason for the changed concentrations of $\mathrm{CCK}$ and gastrin in vagotomised rats is not fully known, but it is likely that a diminished rate of acid secretion and a changed gastrointestinal motility caused by the operation are of importance.

Cholecystokinin was found to be released in response to suckling in lactating rats, which is in accordance with previous data in dogs. ${ }^{13}$ The ratio CCK-8/CCK-33, 39 was in favour of the larger forms in control samples but was changed in favour of CCK-8 in suckling samples. Thus, a tendency of CCK-8 predominance is seen after both feeding and suckling. Plasma concentrations of gastrin were not significantly increased in response to suckling, which is in contrast with previous findings in dogs. ${ }^{13} \mathrm{We}$ have previously hypothesised that suckling induces a reflex activation of the vagal nerve, which leads to a release of several gastrointestinal hormones such as insulin, gastrin, and CCK in lactating animals. ${ }^{13.3}$ As CCK can be released in response to vagal stimulation, ${ }^{34}$ raised plasma concentrations of CCK after suckling may be caused by such an activation of the vagal nerve.

In conclusion, the present study shows a new and specific method for determination of CCK-8 and CCK-33, 39 in rat plasma, based on enzyme digestion of gastrin before HPLC separation and detection by a carboxyl-terminal assay. By this method we have measured raised plasma concentrations of $\mathrm{CCK}$ in response to physiological stimuli such as feeding and suckling in lactating rats.

The present work has been supported by grants from the Swedish Medical Research Council (project nos 05207 and 1010), the Swedish Society of Medicine, the Nordisk Insulinfond, the Swedish Society for Medical Research, and by the Karolinska Institute. 


\section{References}

1 Jorpes JE, Mutt V. Secretin and cholecystokinin (CCK). In: Jorpes JE, Mutt V, eds. Handbook of experimental pharmacology. Berlin: Springer Verlag, 1973: 1-179.

2 Debas HT, Farooq O, Grossman MI. Inhibition of gastric emptying is a physiological action of cholecystokinin. Gastroenterology 1975; 68: 1211-7.

3 Eysselein VE, Böttcher W, Kauffman GL Jr, Walsh JH. Molecular heterogeneity of canine cholecystokinin in portal and peripheral plasma. Regul Pept 1984; 9: 17385.

4 Wolfe MM, Mcguigan JE. Immunochemical characterization of gastrinlike and cholecystokininlike peptides released in dogs in response to a peptone meal. Gastroenterology 1984; 87: 323-34.

5 Maton PN, Selden AC, Chadwick VS. Large and small forms of cholecystokinin in human plasma: measurement using high pressure liquid chromatography and radioimmunoassay. Regul Pept 1982; 4: 251-60.

6 Calam J, Ellis A, Dockray GJ. Identification and measurement of molecular variants of cholecystokinin in duodenal mucosa and plasma. J Clin Invest 1982; 69: 218-25.

7 Lilja P, Wiener I, Inoue K, Fried GM, Greerly GH Jr, Thompson JC. Release of cholecystokinin in reponse to food and intraduodenal fat in pigs dogs and man. Surg Gynecol Obstet 1984; 159: 557-61.

8 Walsh JH, Lamers CB, Valenzuela JE. Cholecystokinin-octapeptide-like immunoreactivity in human plasma. Gastroenterology 1982; 82: 438-44.

9 Izzo RS, Brugge WR, Praissman M. Immunoreactive cholecystokinin in human and rat plasma: correlation of pancreatic secretion in response to CCK. Regul Pept 1984; 9: 21-34.

10 Fölsch UR, Schafmayer A, Ebert R, Becker HD, Creutzfeldt W. Elevated plasma cholecystokinin concentrations in exocrine pancreatic atrophy in the rat. Digestion 1984; 29 : 60-4.

11 Liddle RA, Goldfine ID, Williams JA. Bioassay of plasma cholecystokinin in rats: effects of food, trypsin inhibitor, and alcohol. Gastroenterology 1984; 87: 542-9.

12 Lindén A, Uvnäs-Moberg $\mathrm{K}$. Plasma levels of cholecystokinin, CCK-8 and CCK-33-39 in response to feeding and during pregnancy in dogs. Scand $J$ Gastroenterol 1987; 22: 859-64.

13 Lindén A, Eriksson M, Carlquist M, Uvnäs-Moberg $\mathrm{K}$. Plasma levels of gastrin, somatostatin and cholecystokinin immunoreactivity during pregnancy and lactation in dogs. Gastroenterology 1987; 92: 578-84.

14 Drapeau GR. Cleavage at glutamic acid with Staphylococcal Protease. In: Hirs CHW, Timasheff SN, eds. Methods in enzymology. New York: Academic Press, 1977: 189-91.

15 Nilsson G. Increased plasma gastrin levels in connection with inhibition of gastric acid in response to sham feeding following bulbar perfusion with acid in dogs. Scand J Gastroenterol 1975; 10: 273-7.

16 Rehfeld JF. Immunochemical studies on cholecystokinin. J Biol Chemistry 1978; 253: 4016-21.
17 Rehfeld JF, Holst JJ, Lindkaer Jensen S. The molecular nature of vascularly released cholecystokinin from the isolated perfused porcine duodenum. Regul Pept 1982; 3: 15-28.

18 Rehfeld JF. Immunochemical studies on cholecystokinin. J Biol Chem 1978; 253: 4022-30.

19 Holmquist AL, Walsh JH, Feldman EJ. Immunochemical distinction between rat gastrin and other mammalian gastrins. Gastroenterology 1977; 72 : 1071.

20 Reeve JR Jr, Dimaline R, Shively JE, Hawke D, Chew $\mathrm{P}$, Walsh JH. Unique amino terminal structure of rat little gastrin. Peptides 1981; 2: 453-8.

21 Thompson JC. Gastrointestinal hormones. Austin: University of Texas Press, 1975: 653.

22 Shaffer MH, Agarwal KL, Noyes BE. Rat gastrin's amino acid sequence determined from the nucleotide sequence of the mRNA. Peptides 1982; 3: 693-6.

23 Maton PN, Selden AC, Chadwick. Differential distribution of molecular forms of cholecystokinin in human and porcine small intestinal mucosa. Regul Pept 1984; 8: 9-19.

24 Bacarese-Hamilton AJ, Adrian TE, Bloom SR. Distribution and heterogeneity of immunoreactive cholecystokinin (CCK) in the mucosa of the porcine gastrointestinal tract. Regul Pept 1984; 9: 289-98.

25 Ryder S, Eng J, Straus E, Yalow RS. Alkaline extraction and characterization of cholecystokininimmunoreactivity from rat gut. Gastroenterology 1981; 81: 267-75.

26 Reeve JR Jr, Eysselein V, Walsh JH, Ben-Avram CM, Shively JE. New molecular forms of cholecystokinin. J Biol Chem 1986; 261: 16392-7.

27 Eng J, Du B-H, Pan Y-CE, Chang M, Hulmes JD, Yalow RS. Purification and sequencing of a rat intestinal 22 amino acid C-terminal CCK fragment. Peptides 1984; 5: $1203-6$.

28 Lindén A, Hansen S, Bednar I, Forsberg G, Södersten $P$, Uvnäs-Moberg K. Sexual activity increases plasma concentrations of cholecystokinin octapeptide and offsets hunger in male rats. $J$ Endocrinol 1987; 115: 91-5.

29 Eysselein VE, Eberlein GA, Hesse WH, Singer MV, Goebell H, Reeve JR Jr. Cholecystokinin-58 is the major circulating form of cholecystokinin in blood. J Biol Chem 1987; 262: 214-7.

30 Eysselein VE, Eberlein GA, Hesse WH, Goebell H, Reeve JR Jr. Detection of cholecystokinin-58 (CCK-58) in human blood. Gastroenterology 1987; 92 (5): 1386.

31 Håkanson $R$, Vallgren $S$, Ekelund $M$, Rehfeld JF, Sundler F. The vagus exerts trophic control of the stomach in the rat. Gastroenterology 1984; 86: 2832.

32 Lichtenberger LM. Importance of food in the regulation of gastrin release and formation. Am J Physiol 1982; 243: 429-41.

33 Uvnäs-Moberg K. Release of gastrointestinal peptides in response to vagal activation induced by electrical stimulation, feeding and suckling. J Auton Nerv Syst 1983; 9: 141-55.

34 Cantor P, Holst JJ, Rehfeld JF. Release of cholecystokinin: importance of nervous control. Dig Dis Sci 1984; 29: 155. 